\title{
Growth of $\mathrm{CaF}_{2}$ Buffer on Si Using Low Energy Cluster Beam Deposition Technique and Study of its Properties
}

\author{
S.S. Bhagwat, A.R. Bhangale, J.M. Patil, V.S. Shirodkar, \\ Department of Physics, The Institute of Science, \\ 15, Madame Cama Road, Mumbai - 400 032, India \\ R. Pinto, P.R. Apte and S.P. Pai, \\ SSE Group, Tata Institute of Fundamental Research, \\ Mumbai - 400005 , India
}

Received 4 September 1998. Revised version received 1 February, 1999

\begin{abstract}
Calcium Fluoride buffer lavers were grown on $\mathrm{Si}<100>$ substrates using the low energy cluster beam deposition technique. The films were annealed at various temperatures ranging between $500^{\circ} \mathrm{C}$ and $700^{\circ} \mathrm{C}$. The SEM studies showed that as-deposited films were well oriented along the c-axis and had very smooth surface morphology. The annealed films on the contarary, showed lowering of peak intensities and roughening of the surface. The dielectric constant derived from the $\mathrm{C}-\mathrm{V}$ measurements at $1 \mathrm{MHz}$ were 2.01 and 18 for as-deposited and annealed films respectively.
\end{abstract}

\section{Introduction}

It seems highly probable that High Tc superconducting thin films will have an impact on microelectronics by making it possible to grow low dispersion, high speed dense superconducting interconnects [1]. Another major area where the low-loss properties of the superconductors will potentially have an impact is the devices operating at microwave frequencies [2]. One major obstacle to the above applications is the difficulty to grow high Tc superconducting films on silicon. This arises due to the fact that silicon diffuses into the superconducting film during annealing at elevated temperatures resulting in broad transitions and low critical current densities [3,4]. Hence a class of materials called alkaline earth fluorides, or IIa-fluorides as they are commonly known, have been investigated for its usefulness to overcome this difficulty. These compounds have a low dielectric constant which decreases with decreasing temperature [5]. Moreover, they are chemically stable and structurally compatible with a number of semiconductors viz., Si, GaAs etc [6]. They also have good thermal match owing to their ionic nature [7]. The surface fields in an ionic compound decreases exponentially leading to an interface wherein the bonds across the interface tend to be weak. This allows to overcome the lattice mismatch strain by rearrangement of dislocations.

Various techniques such as sputtering [8], pulsed laser deposition [9] etc., have been employed to obtain good quality buffer layers on silicon. The low energy cluster beam deposition technique, a variant of ionized cluster beam deposition technique, involves formation of clusters of the materials to be deposited which is charged in a specially designed crucible. The material vapours escaping the nozzle of the crucible undergo sudden adiabatic expansion and some of it form clusters. These clusters subsequently move towards the substrate at an ejection velocity and deposit on it. One of the greatest advantages of the low energy cluster beam deposition technique is the absence of electrical charge effect which can influence the nucleation and growth process. 
In this paper, we report on the fabrication of $\mathrm{CaF}_{2}$ buffer layers on Si using the low energy cluster beam deposition technique and study of its dielectric behaviour.

\section{Experimental}

Calcium fluoride (99.99\%) powder was introduced in a specially designed crucible fabricated from high purity electronic grade graphite. The graphite crucible contained a cylindrical cavity of $6 \mathrm{~mm}$ in diameter. The Nozzle diameter (D) was $1 \mathrm{~mm}$ and the thickness (L) of $1 \mathrm{~mm}$ so that the ratio $\mathrm{L} / \mathrm{D}=1$. The ratio $\mathrm{L} / \mathrm{D}$ is preferably kept close to 1 to keep the pressure ratio $\mathrm{Po} / \mathrm{P}$ at a very high value to increase sufficiently the collision rate of the vaporized atoms in the nozzle region. Here Po is the vapor pressure inside the crucible and $\mathrm{P}$ is the vapor pressure outside. Hence the pressure ratio can be increased by decreasing the background pressure to the range of $10^{-7}$ to $10^{-6}$ Torr. The crucible was mounted on a four source turrete and was heated resistively using a tungsten wire. The highest temperature that could be attained inside the crucible was of the order of $1800^{\circ} \mathrm{C}$ and was monitored using a Pt-(Pt)Rh thermocouple positioned in close contact with the crucible. The crucible was fitted inside a chamber which could be evacuated to an ultimate pressure of $10^{-7}$ Torr using an Edwards Co. U.K., turbomolecular pump backed by a rotary pump. The thickness of the film was monitored using a digital thickness monitor, Maxtech Co., USA model MDC 400. More details of the growth procedure are described elsewhere [10].

Silicon $<001>$ substrates used to deposit $\mathrm{CaF}_{2}$ were cleaned using a standard procedure. Before mounting the silicon substrates in the chamber; they were dipped in dil. Nitric acid for a few seconds, washed in distilled water, exposed to alcohol vapours and then dried. After the deposition; films were annealed in a Carbolite Co., U.K, programmable furnace in the temperature range of $550-700^{\circ} \mathrm{C}$. These annealed films as well as the as- deposited films were characterized using JOEI, 8030 X-ray diffractometer and JOEL 840 Scanning Electron Microscope. Capacitance-Voltage (C-V) measurements were carried out by transferring the sam- ple to a cryostat which could be evacuated to $10^{-2}$ Torr during the measurements. The $\mathrm{C}-\mathrm{V}$ characteristics were obtained using Hewlett Packard LCR meter model HP $4824 \mathrm{~A}$ and a bias fixture model No. $16065 \mathrm{~A}$.

\section{Results and Discussion}

Fig. 1 gives the XRD pattern of the powder used for deposition. The intensity peak appearing at $2 \theta=28.30^{\circ}$ corresponds to $\langle 111\rangle$ orientation while that at $47.04^{\circ}$ and $55.78^{\circ}$ corresponds to $<002>$ and $<311>$ orientation respectively. The Characteristic doublet of $<004>$ orientation appears at $68.66^{\circ}$ and $68.90^{\circ}$. Fig. 2 shows the X-ray diffractographs of the films annealed at temperatures ranging from $550^{\circ}$ to $650^{\circ} \mathrm{C}$. Comparison of the XRD pattern of these films with that of the powder shows that only $<001>$ peaks appear prominently indicating that the films are highly $c$-axis oriented. It is evident from the figure 2 that the $<002>$ peak becomes sharper as the annealing temperature increases and is sharpest at $650^{\circ} \mathrm{C}$ beyond which the intensity again decreases. Fig. 3 shows the X-ray diffractograph of the as-deposited films which indicates that the as-deposited film is also $c$-axis oriented. Hence to estimate the level of crystallinity in various films, a full width half maximum (FWHM) was measured for all the films using the $<004>$ peak as standard, it being the most intense peak. It is important to note that the FWHM for as deposited film is minimum to the vahle $0.074^{\circ}$. On the other hand films annealed at different temperatures show a systematic improvement in the crystallinity, going through a maximum at $650^{\circ} \mathrm{C}$. However, the minimum in FWHM for annealed films is still greater in magnitude compared to as- deposited films. This behaviour suggests that the deposition of a material carried out using low energy clusters results into well oriented films. 


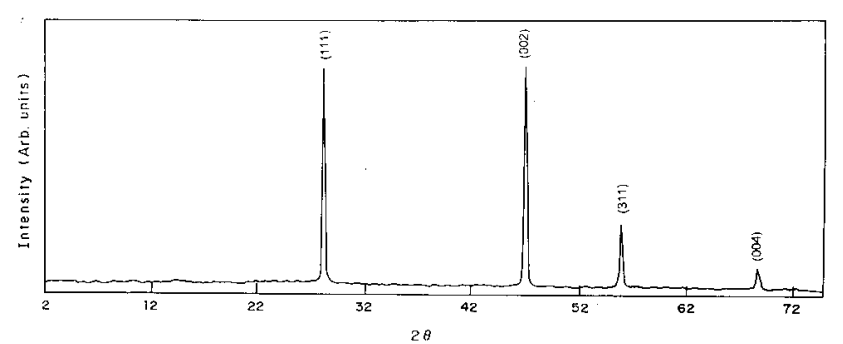

Figure 1. X-Ray diffraction pattern of the $\mathrm{CaF}_{2}$ powder used for the deposition.

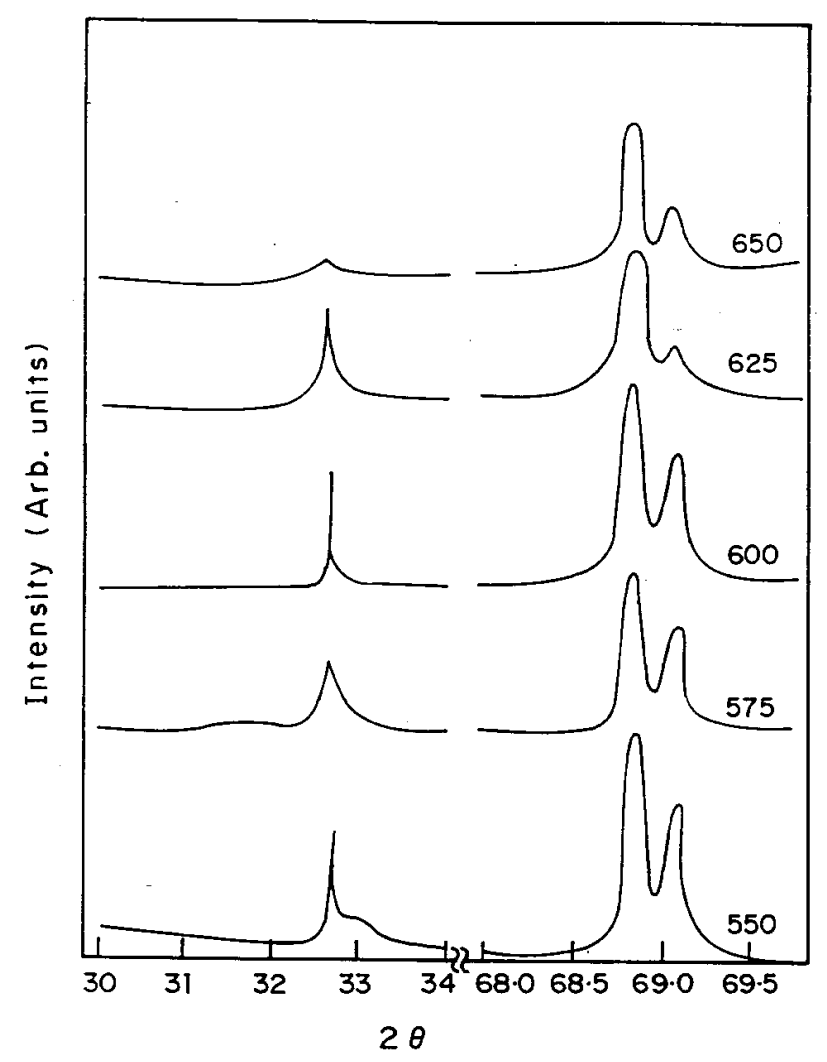

Figure 2. X-ray diffraction pattern of the $\mathrm{CaF}_{2}$ film annealed at temperatures ranging from $550^{\circ}$ to $650^{\circ} \mathrm{C}$.

It has also been observed by Perez et al [11] that the films obtained by low energy cluster beam deposition technique contain large clusters of the host material which do not fragment on the substrate leading to a random substrate "pavement" and orient themselves to a suitable ordering with the substrate. The clusters subsequently diffuse and coalesce with other clusters or would remain as separate entity depending on their size. It is expected therefore, that during post annealing the clusters will gain some kinetic energy (obtained from thermal energy) and hence fragment and thereby the original orientation will be disturbed. However, at lower annealing temperature all the clusters may not fragment and re-orient themselves which would result in the decrease in FWHM. As the annealing temperature increases, more and more clusters fragment and re-orient showing optimum activity at $650^{\circ} \mathrm{C}$. The optimum temperature could be expected to depend on the nature of the substrate and also on the type of material deposited. A further increase in the temperature, beyond the optimum, would be expected to result in surface to film reaction reducing the crystallinity. It was also observed that the substrate temperature attained during the deposition (due to the heat recieved from the crucible source) was close to the optimum annealing temperature.

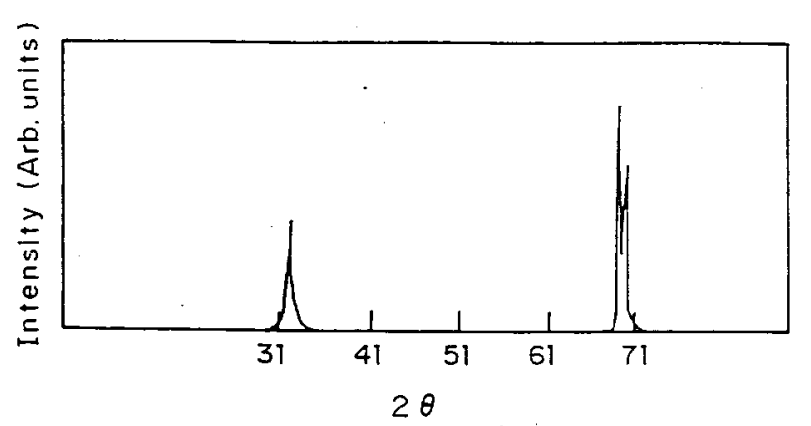

Figure 3. X-ray diffraction pattern of the as-deposited $\mathrm{CaF}_{2}$ film.

To investigate the effect of annealing on the film surface, Scanning Electron Microscopy (SEM) study was carried out for as-deposited film and the films annealed at temperatures $600^{\circ} \mathrm{C}, 650^{\circ} \mathrm{C} ; 700^{\circ} \mathrm{C}$, each for one hour duration. It is seen that the surface of the film alnnealed at $600^{\circ} \mathrm{C}$ (figure $4 \mathrm{a}$ ) is grainy when compared to the surface of the film annealed at $650^{\circ} \mathrm{C}$ (figure $4 \mathrm{~b}$ ) which is the optimum temperature. Fig. 4c gives the SEM picture of the film annealed at $700^{\circ} \mathrm{C}$. It is seen that the film is discontinuous with isolated island formation. The substrate to the film reaction is also evident. However, as-deposited film, figure $4 \mathrm{~d}$ shows a very smooth surface. 


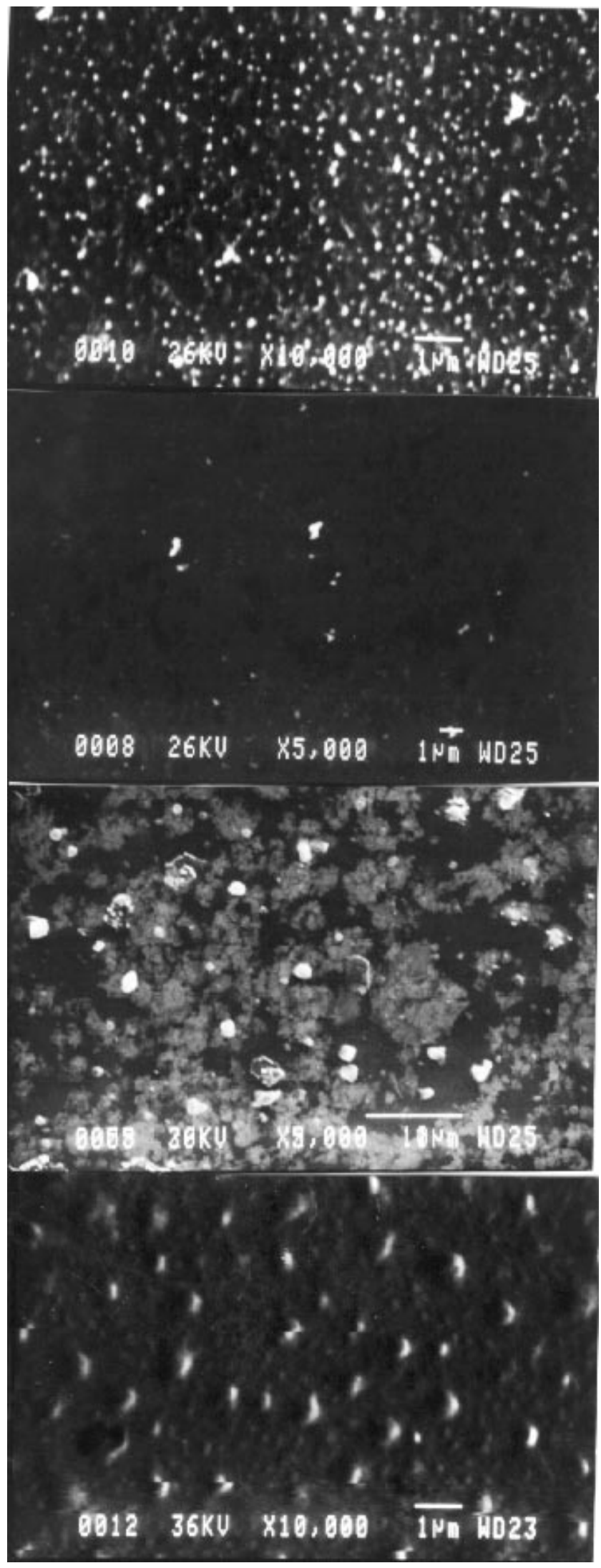

Figure 4. SEM photographs of a) film annealed at $600^{\circ} \mathrm{C}$; b) film annealed at $650^{\circ} \mathrm{C}$; c) film annealed at $700^{\circ} \mathrm{C}$; d) as-deposited film.

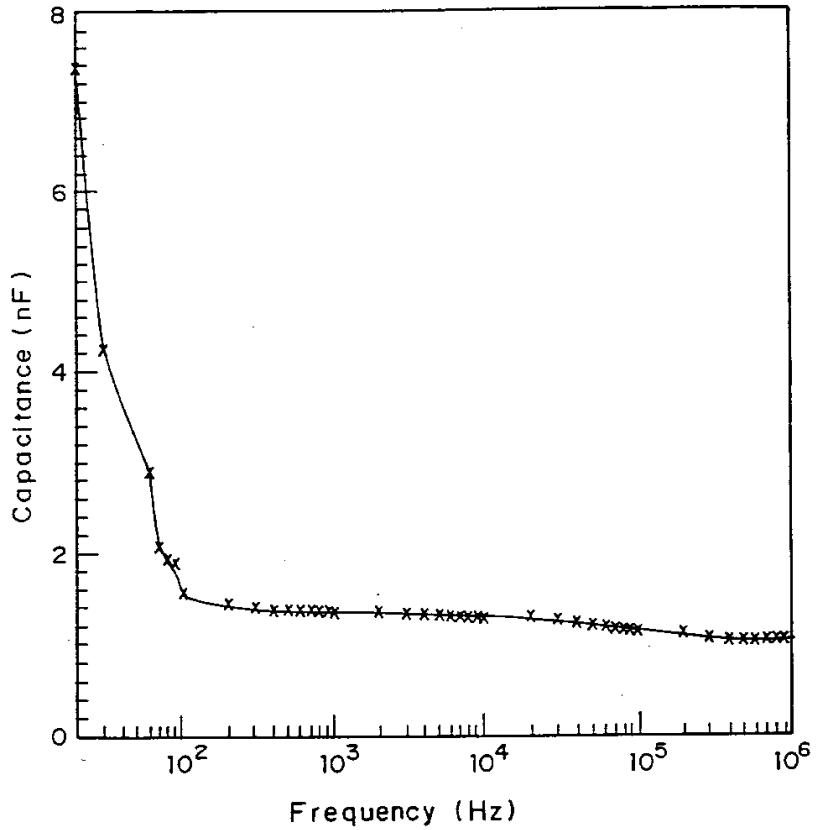

Figure 5. Frequency vs Capacitance plot of the Metal/ $\mathrm{CaF}_{2} /$ Metal structure.

To as certain the usefulness of the $\mathrm{CaF}_{2}$ interlayer for superconductor films used in microwave applications $\mathrm{C}-\mathrm{V}$ measurements were carried out. Fig. 5 shows the frequency $\mathrm{Vs}$ capacitance characteristics of $\mathrm{CaF}_{2}$ films in the form of Metal-Insulator-Semiconductor structure. The capacitance is found to saturate at higher frequencies as expected for a MIS structure. Fig. 6 shows the capacitance-voltage measurements carried out at $1 \mathrm{Mhz}$ frequency for the films annealed at optimum temperature of $650^{\circ} \mathrm{C}$ and the as-deposited film. The total capacitance $\mathrm{C}$ of the MIS diode could be represented [12] as a series combination of the insulator capacitance $C_{i}$ and the semiconductor depletion layers capacitance $C_{j}$. Hence,

$$
C=C_{i} C_{j} /\left(C_{i}+C_{j}\right)
$$

The total capacitance is seen to decrease with the increasing forward voltage while the surface is depleted of charges. When the applied voltage is reversed accumulation of holes at the semiconductor surface takes place. As a result, the total capacitance is close to the insulator capacitance $\epsilon_{i} / d$ where $\epsilon_{i}$ is the dielectric constant of the insulator material and $d$ is the thickness of the insulator material. Hence the maximum capacitance can be considered as the insulator capacitance. Accordingly, the dielectric constant is calculated using the $\mathrm{C}-\mathrm{V}$ plot for the as-deposited films gives the value of the constant as 2.01 which is in good aggrement with the reported values [13]. However the dielectric con- 
stant of the film annealed at optimum temperature is as high as 18 .

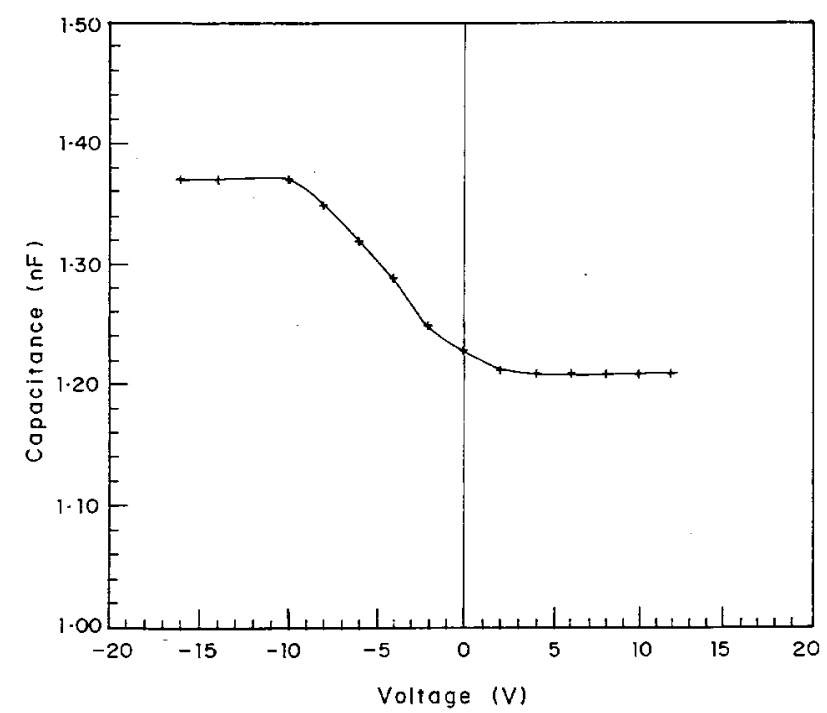

Figure 6. C-V plot of the Metal/ $\mathrm{CaF}_{2} /$ Metal structure at $1 \mathrm{MHz}$.

\section{Conclusion}

Good quality $c$-axis oriented $\mathrm{CaF}_{2}$ buffer layers on $\mathrm{Si}$ were grown using the low energy cluster beam deposition technique. As-deposited film were found to ensure a good surface morphology and better crystallinity than the post annealed films. The dielectric constant of the as-deposted films is of the order of 2.01 which is in close agreement with the reported values.

\section{References}

1. O.W. Kwon, B.W. Langlew, R.F.W. Pease, M.R. Beasley, EEE Electron Devices Lett. EDL-8, 582, (1987).
2. W. Hartwig, C. Passov, Applied Superconductivity, Edited by V.L. Newhouse (Academic Press, New York) Vol ii p542.

3. G. Koren, E.Polturak, B. Fisher, D. Cohen and G. Kimel, Appl. Phys. Lett. 53, 2330 (1988).

4. T. Venkatesan, E.W. Chase, X.D. Wu, A. Inam, C.C. Chang, F.D. Shokoohi, Appl. Phys. Lett. 53, 243 (1988).

5. R.P. Lowndess, J. Phys C. 2, 1595 (1969).

6. H. Ishiwara, T. Asano, Appl. Phys. Lett. 40, 66 (1982).

7. A.N. Tiwari, S. Blunier, H. Zogg, J. Appl. Phys. 71, 10 (1992).

8. Susumu Horita, M. Murakawa, T. Fujiyama, Jpn. J. Appl. Phys. 34, 1942 (1995).

9. J. Bohandy, E. Agostinelli, B.F. Kim, W.J. Green, T.E. Phillips, F.J. Adrian, K. Moorjani, J. Appl. Phys. 65, 4147, (1989).

10. S.S. Bhagwat, A.R. Bhangale, J.M. Patil, V.S. Shirodkar, Ramprasad, N.C. Soni, Proc. Int Con. Vac. Sci and Tech., CAT Indore, Vol 2, pp 345 (1995).

11. A. Perez, P. Melinon, V. Paillard, V. Dupuis, P. Jensen, A. Hoareau, J.P. Perez, J. Tuaillon, M. Broyer, J.L. Vialle, M. Pellarin, J. Lerme, Nanostructured Materials, 34, 43 (1995).

12. S.M. Sze, Physics of Semiconductor Devices, 2nd ed., pp 371 (1981).

13. Sui-Wai Charl, E.W. Chase, B.J. Wilkenns, D.L. Hart, Appl. Phys. Lett. 54, 20 (1989). 